\title{
Bone Sialoprotein Gene Transfer to Periodontal Ligament Cells May Not Be Sufficient to Promote Mineralization In Vitro or In Vivo
}

\author{
Sema S. Hakki, ${ }^{*}$ Dian Wang, ${ }^{\dagger}$ Renny T. Franceschi, ${ }^{\dagger}$ and Martha J. Somerman ${ }^{\S}$
}

Background: To improve regenerative therapies, it is important to understand the cells and factors modulating periodontal tissues. Our group has focused on bone sialoprotein (BSP), a mineralized tissueselective protein considered to be involved in the initiation of cementogenesis and osteogenesis. In this study, we examined whether gene transfer of BSP into periodontal ligament (PDL) cells would result in an increased ability of PDL cells to promote mineralization in vitro and in vivo.

Methods: PDL cells obtained from CD-1 mice were immortalized using simian virus (SV) 40 large T antigen (TAg) and designated SV-PDL cells. SV-PDL cells were infected in vitro with LacZ gene-expressing control adenovirus vector. A 1,000 plaque-forming unit (pfu) titer was selected (based on X-gal staining) and cells were infected with mouse BSP-expressing replication-deficient adenoviral vector to determine the mRNA expression and protein level of BSP. Total RNA was isolated from cells on days 2, 4, and 6. Media were obtained on days 3, 5, and 7 for protein determination. Northern blot analysis was performed for mRNA expression and Western blot analysis for protein expression. To test the effect of BSP gene transfer on the mineralization of PDL cells, in vitro (von Kossa) and in vivo (severe combined immunodeficiency [SCID] mice) experiments were performed.

Results: Under normal conditions, PDL cells do not express BSP transcripts and do not promote significant mineralization. SV-PDL cells infected with a BSP viral vector expressed and secreted substantial levels of BSP as confirmed by Northern and Western blot analysis. BSP mRNA and protein levels were strong on day 2 and still apparent on day 6 , although not as great. However, no mineral nodule formation was noted either in vitro or in vivo.

Conclusions: Although BSP is an important and necessary protein for mineralization, it may not be sufficient for promoting mineralization without the addition or removal of other factors. Further studies will help to clarify the specific factors required for promoting mineralization, a required step for designing predictable periodontal regenerative therapies. J Periodontol 2006;77:167-173.

\section{KEY WORDS}

Bone sialoprotein; gene therapy; periodontal ligament cells; regeneration.

\footnotetext{
* Department of Periodontology, Faculty of Dentistry, Selcuk University, Konya, Turkey.

$\dagger$ Department of Periodontics/Prevention/Geriatrics, Faculty of Dentistry, University of Michigan,

Ann Arbor, MI.

‡ Department of Biological Chemistry, Medical School, University of Michigan.

$\S$ School of Dentistry, University of Washington, Seattle, WA.
}

Tissue engineering is an exciting field that aims to recreate functional, healthy tissues and organs to replace diseased ones. Gene therapy is becoming a reality, and it is a particularly attractive approach for promoting wound healing. The potential use of gene therapy to treat human diseases increases with the concomitant development of various physical, chemical, and biologic methods to deliver genes to mammalian cells and with our rapidly expanding knowledge of the human genome. The adenovirus has advantages as a vector system in gene therapy due to its ease of producing a high titer recombinant virus and high transduction efficiency, as well as its ability to transfer the gene of interest, even into non-dividing cells. ${ }^{1}$

The essential purpose of regenerative periodontal therapies is to achieve reconstruction of the periodontal attachment apparatus, i.e., new bone and cementum and a functional periodontal ligament (PDL), lost due to periodontal disease. Research to determine appropriate treatment modalities to

doi: 10.1902/jop.2006.050057 
promote the regeneration of lost periodontal tissues has grown rapidly. Current periodontal regenerative therapies include guided tissue regeneration using membranes (bioabsorbable or non-resorbable), grafts (autograft or allograft) and some root conditioning agents (citric acid or tetracycline), and growth and differentiation factors using a variety of delivery systems. ${ }^{2,3}$

In the last decade, studies have focused on understanding the mechanisms regulating wound repair and regeneration at the cellular and molecular level. Periodontal tissue regeneration is a complex process that invokes the proliferation of specific progenitor cells, repopulation of appropriate cells at the healing site followed by maturation and secretion of an extracellular matrix as required for formation of mineralized tissue, i.e., bone and cementum, and development of a functional PDL. To obtain more predictable regenerative therapies, more recent research has been targeted at identifying specific cells and factors functioning during the regeneration of periodontal tissues. As one tool for determining the required factors, our laboratory has designed experiments that include infecting cells with putative regenerative genes using adenovirus constructs; an attractive candidate for such studies is bone sialoprotein (BSP). ${ }^{4}$

BSP is a major non-collagenous protein that has a highly restricted expression to mineralized skeletal tissues and is expressed during initial stages of bone and cementum formation. BSP, an early marker of osteoblast differentiation, has been implicated in the nucleation of hydroxyapatite during bone formation. ${ }^{5-8}$ In vitro BSP mRNA expression increases during periods of matrix mineralization. ${ }^{9}$

The purpose of this study was to test whether gene transfer of BSP into PDL cells would result in an increased ability of PDL cells to promote mineralization in vitro and in vivo.

\section{MATERIALS AND METHODS}

\section{Cell Culture}

In these experiments, mouse PDL cells transformed with simian virus 40 (SV 40) T antigen-containing virus were used. ${ }^{10}$ SV-PDL cells were plated at a density of 40,000 cells $/ \mathrm{cm}^{2}$. Cells were infected with adenovirus constructed with mouse BSP (m-BSP) gene. As a first step, different titers of virus were tested for optimization of infection with LacZ virus. The cells were infected with LacZ virus at 125, 250, 500, 1,000 , and 2,000 plaque-forming units (pfu) per cells. $\mathrm{X}$-gal staining was performed to confirm infection of cells. For optimum infection, 1,000 pfu per cell was selected based on X-gal staining. ${ }^{11}$ Similar results were obtained from three separate experiments.

\section{Morphology}

Images of infected PDL cells were examined visually using a phase contrast microscopell on days 2,4 , and 6 .

\section{Northern Blot Analysis}

SV-PDL cells were plated at a density of 40,000 cells/ $\mathrm{cm}^{2}$. Total RNA was isolated with reagent ${ }^{\text {Il }}$ on days 2 , 4 , and 6 after infection. RNA concentration was quantified by a spectrophotometer. RNA ( $5 \mu \mathrm{g}$ ) was denatured, fractionated on $6 \%$ formaldehyde and $1.2 \%$ agarose gel, transferred to a nylon membrane, and cross-linked by ultraviolet radiation (UV). Blots were hybridized with random primed ${ }^{32} \mathrm{P}$-labeled probes and exposed to film ${ }^{\#}$ with intensifying screens at $-70^{\circ} \mathrm{C}$ for 24 to 48 hours. Probes used for Northern blots were as follows: BSP $=$ M-BSP consisting of $1 \mathrm{~kb}$ of mouse cDNA in polymerase chain reaction (PCR) II $^{12}$ (a gift from Drs. M. Young and L. Fisher, National Institute of Dental and Craniofacial Research/National Institutes of Health [NIDCR/NIH], Bethesda, MD); osteopontin $(\mathrm{OPN})=$ MOP-3 consisting of $1 \mathrm{~kb}$ of mouse OPN cDNA in PCR II ${ }^{13}$ (a gift from Drs. M. Young and L. Fisher, NIDCR/NIH); osteocalcin $(\mathrm{OCN})=400$ bp of mouse OCN cDNA originally cloned into pSP65 cloning vector and transferred to plasmid**,14 (obtained from Dr. J. Wozney, Genetic Institute, Cambridge, MA); matrix-gla protein (MGP) $=398$ bp mouse MGP cDNA in plasmid ${ }^{\dagger \dagger}$ (obtained from Dr. Gerard Karsenty, Department of Molecular and Human Genetics, Baylor College of Medicine, Houston, TX). ${ }^{15}$ Experiments were carried out twice.

\section{Western Blot Analysis}

Conditioned medium was collected on days 3, 5, and 7 after infection for Western blot analyses. Conditioned medium was prepared by incubating cells in serum-free Dulbecco's modified Eagle's medium (DMEM) for 24 hours. Dialyzed and lyophilized samples were dissolved in $1 \times$ sodium dodecyl sulfatepolyacrylamide gel electrophoresis (SDS-PAGE) loading buffer. Aliquots containing $25 \mu \mathrm{g}$ protein were fractionated by SDS-PAGE on $10 \%$ gels and electrophoretically transferred to a nitrocellulose membrane. First antibody (rabbit immunoglobulin [Ig] G) was used at a dilution of $1: 2,000$ in $10 \mathrm{mM}$ Tris- $\mathrm{HCl}$, $0.15 \mathrm{M} \mathrm{NaCl}$, and $0.2 \%$ Tween 20 (TBST) $+0.5 \%$ milk. Second antibody (goat anti-rabbit IgG) was used at a dilution of $1: 20,000$ in TBST $+0.5 \%$ milk. Immunoreactivity was determined using the enhanced chemiluminescence reaction. ${ }^{16}$

\footnotetext{
Axiovert 35, Carl Zeiss, Thornwood, NY

If Trizol, Invitrogen, Gaithersburg, MD.

\# Kodak X-Omat or Biomax, Kodak, Rochester, NY.

** Bluescript SK, Stratagene, La Jolla, CA.

$\dagger \dagger$ Bluescript, Stratagene.
} 


\section{Mineralization}

In vitro mineralization. The in vitro mineralization assay was performed on SV-PDL cells infected with sense and antisense BSP gene. Cells were plated at $4 \times 10^{5}$ cells $/ \mathrm{cm}^{2}$ in 24 -well plates in DMEM containing $10 \%$ FBS. After 24 hours, cells were infected and then maintained with DMEM, supplemented with ascorbic acid (AA, $50 \mu \mathrm{g} / \mathrm{ml}$ ), and $10 \mathrm{mM} \beta$ glycerophosphate. ${ }^{17,18}$ Biomineralization of PDL cells was determined on day 10 by von Kossa staining in vitro. Experiments were performed two times.

Ex vivo biomineralization. To determine whether biomineralization formation occurred in vivo, the procedures described below were approved by the University of Michigan Committee and the Unit for Laboratory Animal Medicine (ULAM) and were compliant with state and federal laws as well as the guiding principles for the Use and Care of Animals. Immunocompromised mice (severe combined immunodeficiency [SCID] mice) $)^{\ddagger \ddagger}$ were used to serve as subcutaneous transplant recipients to avoid host rejection of cell transplants. Previous studies have displayed that this SCID mice model provides an excellent environment for supporting formation of mineralized tissues by different cell types including mouse osteoblastic cells (MC3T3E1) and cementoblasts. ${ }^{19}$ Transplant vehicles were prepared by soaking $3 \times 3 \mathrm{~mm}$ squares of a mixture of hydroxyapatite and collagen $\S \S$ in DMEM containing $10 \%$ FBS for 40 to 45 minutes, followed by compression between filter paper to remove air. SV-PDL cells were infected with sense and antisense BSP gene containing adenovirus (1,000 pfu) and trypsinized, pelleted, and resuspended at $3.0 \times 10^{6}$ cells $/ \mathrm{ml}$. Sponges ${ }^{\| l}$ alone and untreated SV-PDL cells served as negative controls. MC3T3-E1 clone 4 cells served as positive control. The compressed sponges were allowed to absorb the cell suspension by capillary action and held at $37^{\circ} \mathrm{C}$ until implantation. SCID mice were anesthetized using methoxyflurane. . $^{\mid \mathbb{f}}$ Two midsagittal incisions were made, cell-seeded sponges were placed into the dorsum of SCID mice, and the sites were stapled closed. Six SCID mice were employed for two different time periods. Implants were taken at 4 and 6 weeks following surgery. Samples were fixed using $10 \%$ neutral buffered formalin and embedded in paraffin. Five-micron sections were prepared and stained with hematoxylin and eosin.

\section{RESULTS}

\section{$X$-Gal Staining (Fig.1)}

To determine the efficiency of adenovirus transduction, SV-PDL cells were treated with an optimal titer of $1,000 \mathrm{pfu} / \mathrm{cell}$ of LacZ virus and stained with X-gal substrate. Nearly $100 \%$ of cells stained positive at all time points examined, indicating that most cells were transduced at this virus titer.

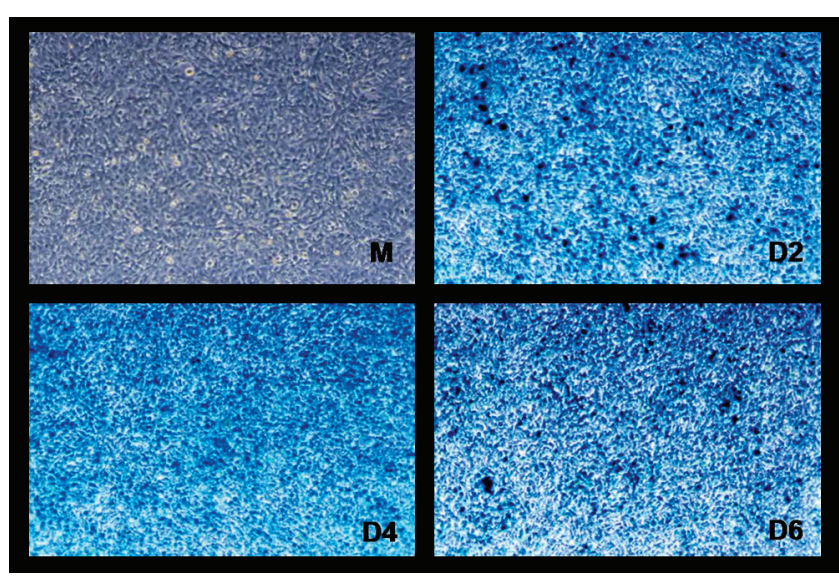

Figure I.

X-gal staining of PDL cells on days 2 (D2), 4 (D4), and 6 (D6) after infection. Cells were infected with 1,000 pfu adenovirus titers.

Note blue-stained cells at all time periods. ( $M=$ mock group; original magnification $\times 10$.)

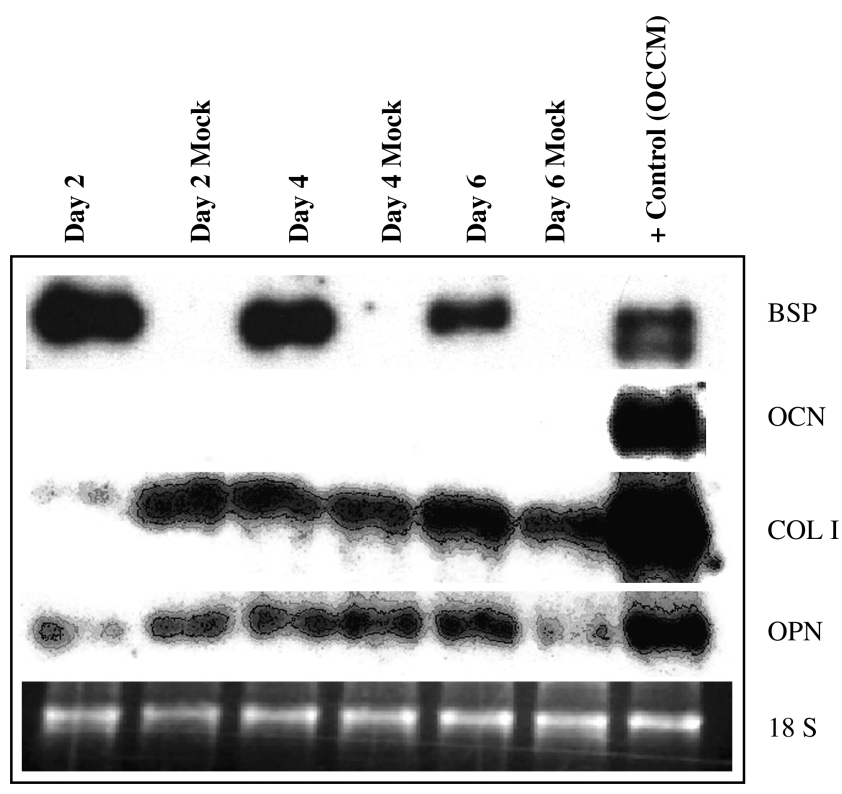

Figure 2.

BSP, OCN, COL I, and OPN gene mRNA expression in PDL cells on days 2, 4, and 6 after infection. Immortalized murine cementoblasts (OCCM) were used as a positive control. BSP mRNA expression was observed only in infected cells on days 2, 4, and 6. The OCN transcript was not noted at any time period. Note inhibited COL I and OPN mRNA expression in infected cells on day 2 and increased OPN transcript in infected SV-PDL cells on day 6.

\section{Northern Analysis (Figs. 2 and 3)}

The PDL cells used here did not express BSP transcripts or promote significant mineralization under

キ C. B-17 SCID, Taconic, Germantown, NY.

$\S \S$ Collagraft, Zimmer, Warsaw, IN.

||| Collagraft, Zimmer.

ขึ Mallincrodt Veterinary, Mundelein, IL. 


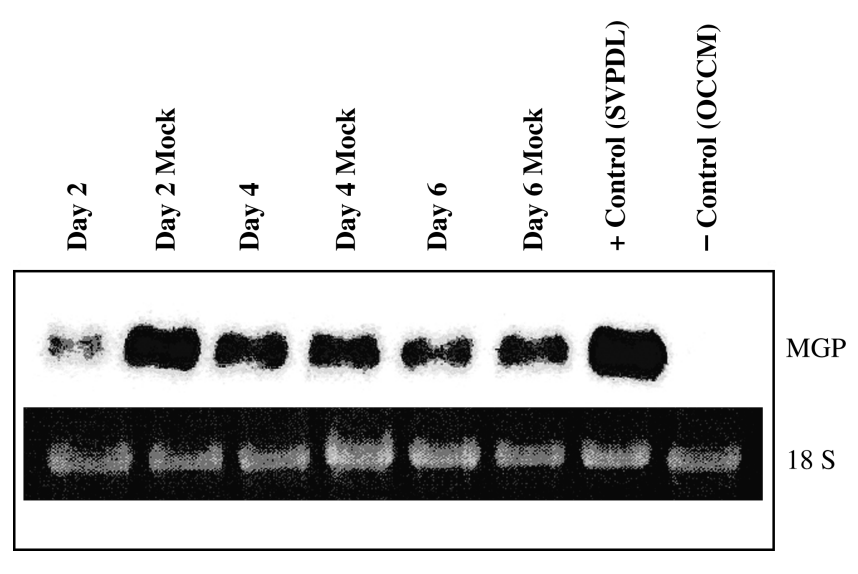

Figure 3.

MGP gene mRNA expression in PDL cells on days 2, 4, and 6 after infection. SV-PDL cells were used as a positive control. OCCM cells were used as a negative control. Note decreased MGP transcript in infected SV-PDL cells on day 2.

normal conditions. ${ }^{10}$ SV-PDL cells infected with a BSP adenoviral vector expressed transcripts for BSP as detected by Northern blot analysis. BSP mRNA expression was strong on day 2 and still apparent on day 6 but not the same as earlier time periods. In concert with BSP infections, changes in mRNA levels were noted for OPN, MGP, and type I collagen (COL I). Decreased expression of mRNA for COL I (Fig. 2) and MGP (Fig. 3) was noted on day 2 in BSP-infected SVPDL cells versus uninfected cells. Increased OPN mRNA expression was noted at day 6 only (Fig. 2). OCN mRNA was not detected in either infected or uninfected cells. These results were reproduced in three separate experiments with a representative result shown here.

\section{Western Analysis (Fig. 4)}

Western blot analysis was performed to confirm the protein levels of BSP. A prominent BSP protein expression was noted on day 3 with decreasing levels on days 5 and 7 . These results were reproduced in three separate experiments.

\section{Mineralization}

The above results clearly show that BSP-gene transfer was performed successfully, resulting in expression of BSP mRNA and protein. Although protein expression persisted for up to 7 days, no mineralized nodule formation was observed when cultures were stained by the von Kossa method. In contrast, MC3T3-E1 osteoblast cells expressing comparable levels of BSP strongly mineralized under these conditions. Also, mineral nodule formation was not observed in vivo at 4 and 6 weeks in the SCID-mice model (data not shown).

\section{DISCUSSION}

The delivery of growth/differentiation factors directly or using gene therapy has become an attractive

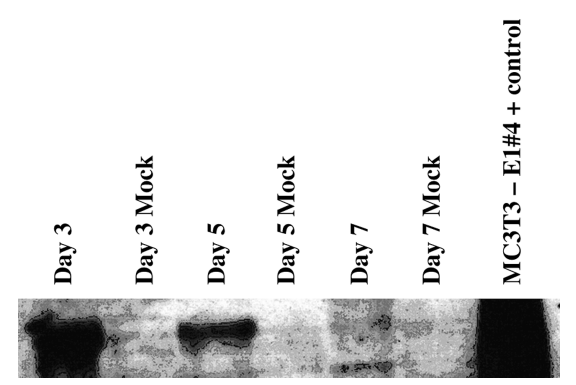

Figure 4.

BSP protein expression in PDL cells on days 3, 5, and 7 after infection, following Western blotting. MC3T3-EI (clone \#4) cells were used as a positive control.

approach for defining the factors required for regenerating periodontal tissues. ${ }^{20-22}$ Extracellular matrix proteins play key roles in controlling the activities of osteoblasts and osteoclasts in bone remodeling. These bone-specific extracellular matrix proteins contain amino acid sequences that mediate cell adhesion. Mineralization of extracellular matrix is a complex process that is believed to involve both hydroxyapatite-nucleating and modulating non-collagenous proteins. In mineralized tissue, hydroxyapatite nucleation is mediated by an anionic phosphoprotein, whereas type I collagen acts a structural matrix. It was believed that BSP is the most likely candidate to regulate nucleation. ${ }^{4,6}$ Non-collagenous proteins associated with mineralized tissues play to regulate nucleation important roles in regulating the behavior of cells within the bone environment. BSP, a mineralized tissue-linked protein, comprises $15 \%$ of the total non-collagenous proteins in bone and has been shown to bind to collagen, calcium, and hydroxyapatite. ${ }^{23,24}$ BSP is expressed by differentiated osteoblasts and appears to function in the initial mineralization and in the remodeling process of bone. ${ }^{25}$ Normally, BSP expression is limited exclusively to mineralized connective tissues, and its expression is localized in bone formation areas. Transfection of BSP into non-mineralizing MC3T3-E1 osteoblast subclones was shown to restore their ability to form mineral deposits. ${ }^{26}$ In the genetically engineered osteosarcoma cell line (K8), overexpression of BSP resulted in an increase in mineral formation in vitro. ${ }^{27}$ Hunter et al. ${ }^{28}$ combined the putative collagen-binding site of mouse decorin with one of two putative hydroxyapatite nucleating sites of pig BSP. Chimeric protein induced the formation of hydroxyapatite crystals in a steadystate agarose gel system and bound with high affinity to fibrillar type I collagen. The addition of chimeric protein to collagen gels perfused with low concentrations of calcium and phosphate resulted in the deposition of large, apparently needle-shaped HA crystals on the surface of collagen fibrils. These findings suggest that 
the BSP-decorin chimeric protein could be capable of inducing the mineralization of collagen in vivo. ${ }^{28}$ Based on this information, BSP is likely to be involved with early mineral deposition. Hydroxyapatite formation can be induced by nucleating proteins such as BSP. ${ }^{7,24,28}$ Hence, it has been suggested that BSP gene therapy or strategies where BSP is delivered to the local area may be available approaches for restoration of destroyed periodontal structures including bone, periodontal ligament, and tooth root cementum.

To determine whether PDL cells that normally do not promote mineral formation would be able to do so, SV-PDL cells were infected with mouse BSPexpressing replication-deficient adenoviral vector. Although the data presented demonstrate successful expression of BSP by PDL cells, these cells were not able to promote mineral nodule formation either in vitro or in vivo. There are several explanations for this finding. Possible reasons why BSP gene therapy did not promote mineralization include the following: 1) insufficient levels of BSP protein in the local area, and/or duration of expression was insufficient; 2) other proteins/factors expressed by PDL cells that are negative regulators of mineralization, e.g., MGP and OPN, block BSP activity; and 3) BSP alone is insufficient for promoting mineral formation.

To our knowledge, this is the first report using BSP gene transfer to promote regeneration of mineralized tissues; hence, the response of SV-PDL cells to BSP gene transduction is noteworthy. Our data suggest that short-term delivery of BSP alone to a local site may be insufficient for promoting mineral formation.

Mineralization of the extracellular matrix in periodontal structures is a complex process that is believed to involve both hydroxyapatite-nucleating and -modulating non-collagenous proteins. MGP is a mineral-binding protein synthesized by cells that produce an uncalcified extracellular matrix. Mice that lack MGP develop to term but die within 2 months as a result of arterial calcification. ${ }^{15}$ MGP-deficient mice additionally exhibit inappropriate calcification of cartilage, including the growth plate, resulting in short stature, osteopenia, and fractures. ${ }^{29} \mathrm{OPN}$ is likely to play a role in the early formative stages of osteogenesis by facilitating the attachment of osteoblasts to the extracellular matrix, and it is also involved in attachment of osteoclasts during bone resorption. ${ }^{30}$ Researchers reported that there is a strong association between increased MGP and OPN mRNA expressions and inhibition of mineralization in cementoblasts and MC3T3E1 murine preosteoblasts. ${ }^{31,32}$ Although decreased MGP and OPN mRNA expressions were noted in the infected cells on day 2, we did not observe mineralization in SV-PDL cells. Variations in the short-term expression of these proteins may not be sufficient to affect mineralization of cells.
Although the SV-PDL cells were induced for BSP mRNA expression with viral infections, cells did not express OCN transcripts, which are known to be an early marker for cells undergoing mineralization and are thought to play a role in bone remodeling. ${ }^{33}$ Hence, the results from this study revealed that transient BSP expression is not sufficient for promoting mineral formation, and further studies are required to obtain mineralization of non-mineralized tissues and to understand the complex mechanisms and interactions of mineralization.

BSP exhibits a specific interaction with type I collagen, and it was demonstrated that there is a high binding affinity between these two proteins. In fetal calvarial organ culture and mineralizing bone cell cultures, a fraction of newly synthesized BSP becomes tightly associated with collagen and can be released only by collagenase digestion. 5,34,35 The binding of BSP to collagen is thought to be important for the initiation of bone mineralization and in the adhesion of bone cells to the mineralized matrix. ${ }^{36}$ Type I collagen acts as a structural matrix, whereas hydroxyapatite nucleation is mediated by an anionic phosphoprotein, i.e., BSP. ${ }^{37}$ In our mRNA expression experiments, decreased collagen type I mRNA was noted on day 2 . The lack of mineralization in vitro and in vivo may be attributed to decreased collagen-type I transcript and insufficient binding with each other in SV-PDL cells for early stages.

Other groups have reported some success in using gene therapy to promote periodontal regeneration. Giannobile et al. ${ }^{38}$ and Jin et al. ${ }^{22}$ transduced cementoblasts with platelet-derived growth factor-A (PDGFA) encoding adenovirus (Ad), and their results demonstrated that gene delivery of PDGF stimulates cementoblast activity that is sustained above that of recombinant human (rh)-PDGF-A application. In addition, Giannobile et al. ${ }^{38}$ and Jin et al. ${ }^{22}$ reported that the use of gene therapy as a mode of growth factor delivery provides a novel approach for periodontal engineering. Anusaksathien et al. ${ }^{39}$ investigated the effect of sustained PDGF gene transfer on cementum formation in an ex vivo ectopic biomineralization model, and their results showed that continuous exposure to PDGF-A had an inhibitory effect on cementogenesis perhaps via upregulation of OPN. The findings of the study by Anusaksathien et al. ${ }^{39}$ suggested that PDGF is required for mineral neogenesis, but continuous exogenous delivery of PDGF-A may delay mineral formation induced by cementoblasts.

Rutherford et al. ${ }^{40,41}$ have shown that fibroblasts transduced ex vivo by bone morphogenetic protein (BMP)-7 cDNA delivered by recombinant adenoviruses induce bone formation and convert to osteoblasts upon implantation in vivo. They also found that BMP-7-transduced human oral keratinocyte cells 
formed ectopic bone as well. ${ }^{40,41} \mathrm{Jin}$ et al. ${ }^{42}$ used ex vivo BMP-7 gene transfer with adenoviral vector in dermal fibroblasts to stimulate tissue engineering of alveolar bone wounds. Their data demonstrated that Ad-BMP-7 gene delivery could promote cementogenesis and osteogenesis ${ }^{42}$ and was the first successful evidence of periodontal tissue engineering employing ex vivo gene transfer of BMPs.

Additional investigations are needed to determine the specific gene or genes required for promoting mineralization. Possible directions for future studies include the use of retroviral vectors and/or stable transfection to give sustained constitutive BSP expression or addition of accessory factors. Alternatively, regenerative strategies can be envisioned in which an early proliferative signal, i.e., PDGF or fibroblast growth factor (FGF), under the control of an adenovirus, is followed by differentiation signals such as BMPs under the control of an inducible promoter. ${ }^{43,44}$ Information obtained from these studies will enable us to design more predictable regenerative therapies by defining the specific factors needed to promote regeneration.

\section{ACKNOWLEDGMENTS}

This study was supported by the Scientific and Technical Research Council of Turkey (TUBITAK/BAYG) and the National Institute of Dental and Craniofacial Research (grant DE09532).

\section{REFERENCES}

1. Prince HM. Gene transfer: A review of methods and applications. Pathology 1998;30:335-347.

2. Giannobile WV, Meraw SJ. Periodontal Applications. Methods of Tissue Engineering. San Diego: Academic Press; 2001:1205-1215.

3. Needleman I, Tucker R, Giedrys-Leeper E, Worthington $\mathrm{H}$. Guided tissue regeneration for periodontal intrabony defects - A Cochrane Systematic Review. Periodontol 2000 2005;37:106-123.

4. Tye CE, Rattray KR, Warner KJ, et al. Delineation of the hydroxyapatite-nucleating domains of bone sialoprotein. J Biol Chem 2003;278:7949-7955.

5. Hunter GK, Goldberg HA. Modulation of crystal formation by bone phosphoproteins: Role of glutamic acid-rich sequences in the nucleation of hydroxyapatite by bone sialoprotein. Biochem $J$ 1994;302: 175-179.

6. Goldberg HA, Warner KJ, Stillman MJ, Hunter GK. Determination of the hydroxyapatite-nucleating region of bone sialoprotein. Connect Tissue Res 1996;35: 385-392.

7. Harris NL, Rattray KR, Tye CE, et al. Functional analysis of bone sialoprotein: Identification of the hydroxyapatite-nucleating and cell-binding domains by recombinant peptide expression and site-directed mutagenesis. Bone 2000;27:795-802.

8. Marom R, Shur I, Solomon R, Benayahu D. Characterization of adhesion and differentiation markers of osteogenic marrow stromal cells. J Cell Physiol 2005; 202:41-48.

9. Yang R, Gerstenfeld LC. Structural analysis and characterization of tissue and hormonal responsive expression of the avian bone sialoprotein (BSP) gene. J Cell Biochem 1997;64:77-93.

10. D'Errico JA, Ouyang H, Berry JE, et al. Immortalized cementoblasts and periodontal ligament cells in culture. Bone 1999;25:39-47.

11. Okubo Y, Bessho K, Fujimura K, lizuka T, Miyatake S. Expression of bone morphogenetic protein-2 via adenoviral vector in $\mathrm{C} 2 \mathrm{C} 12$ myoblasts induces differentiation into the osteoblast lineage. Biochem Biophys Res Commun 1999;262:739-743.

12. Young MF, Ibaraki K, Kerr JM, Lyu MS, Kozak CA. Murine bone sialoprotein (BSP) cDNA cloning, mRNA expression, and genetic mapping. Mamm Genome 1994;5:108-111.

13. Young MF, Kerr JM, Termine JD, et al. cDNA cloning, mRNA distribution and heterogeneity, chromosomal location, and RFLP analysis of human osteopontin (OPN). Genomics 1990;7:491-502.

14. Celeste AJ, Rosen V, Buecker JL, Kriz R, Wang EA, Wozney JM. Isolation of the human gene for bone gla protein utilizing mouse and rat CDNA clones. EMBO $J$ 1986;5:1885-1890.

15. Luo G, Ducy P, Mckee MD, et al. Spontaneous calcification of arteries and cartilage in mice lacking matrix gla protein. Nature 1997;386:78-81.

16. Lou J, Xu F, Merkel K, Manske P. Gene therapy: Adenovirus-mediated human bone morphogenetic protein-2 gene transfer induces mesenchymal progenitor cell proliferation and differentiation in vitro and bone formation in vivo. J Orthop Res 1999;17:43-50.

17. Franceschi RT, Iyer BS, Chi Y. Effects of ascorbic acid on collagen matrix formation and osteoblast differentiation in murine MC3T3-E1 cells. J Bone Miner Res 1994;9:843-854

18. Hakki SS, Berry JE, Somerman MJ. The effects of enamel matrix protein derivative on follicle cells in vitro. J Periodontol 2001;72:679-687.

19. Tokiyasu Y, Saygin NE, Somerman MJ. Enamel factors regulate expression of genes associated with cementoblasts. J Periodontol 2000;71:1829-1839.

20. Zhu Z, Lee CS, Tejeda KM, Giannobile WV. Gene transfer and expression of platelet-derived growth factors modulate periodontal cellular activity. J Dent Res 2001;80:892-897.

21. Chen QP, Giannobile WV. Adenoviral gene transfer of PDGF downregulates gas gene product PDGFalphaR and prolongs ERK and Akt/PKB activation. Am J Physiol Cell Physiol 2002;282:C538-544.

22. Jin $Q$, Anusaksathien $O$, Webb SA, Printz MA, Giannobile WV. Engineering of tooth-supporting structures by delivery of PDGF gene therapy vectors. Mol Ther 2004;9:519-526.

23. Wuttke M, Muller S, Nitsche DP, Paulsson M, Hanisch FG, Maurer P. Structural characterization of human recombinant and bone-derived bone sialoprotein. Functional implications for cell attachment and hydroxyapatite binding. J Biol Chem 2001;276:3683936848.

24. Goldberg HA, Warner KJ, Li MC, Hunter GK. Binding of bone sialoprotein, osteopontin and synthetic polypeptides to hydroxyapatite. Connect Tissue Res 2001; 42:25-37. 
25. Ganss B, Kim RH, Sodek J. Bone sialoprotein. Crit Rev Oral Biol Med 1999;10:79-98.

26. Wang D, Christensen K, Chawla K, Xiao G, Krebsbach $\mathrm{PH}$, Franceschi RT. Isolation and characterization of MC3T3-E1 preosteoblast subclones with distinct in vitro and in vivo differentiation/mineralization potential. J Bone Miner Res 1999;14:893-903.

27. Gerstenfeld LC, Uporova T, Schmidt J, et al. Osteogenic potential of murine osteosarcoma cells: Comparison of bone-specific gene expression in in vitro and in vivo conditions. Lab Invest 1996;74:895-906.

28. Hunter GK, Poitras MS, Underhill TM, Grynpas MD, Goldberg HA. Induction of collagen mineralization by a bone sialoprotein-decorin chimeric protein. J Biomed Mater Res 2001;55:496-502.

29. Luo G, D'Souza R, Hogue D, Karsenty G. The matrix gla protein is a marker of the chondrogenesis cell lineage during mouse development. J Bone Miner Res 1995; 10:325-334.

30. Noda M, Denhardt D. Osteopontin. In: Bilezikian JP, Raisz LG, Rodan GA, eds. Principles of Bone Biology, 2nd ed., vol. 1. San Diego: Academic Press; 2002: 239-250.

31. Hakki SS, Nohutcu RM, Hakki EE, Berry JE, Akkaya MS, Somerman MJ. Dexamethasone and basic-fibroblast growth factor regulate markers of mineralization in cementoblasts in vitro. $J$ Periodontol 2005;76:15501558.

32. Gopalakrishnan R, Ouyang H, Somerman MJ, McCauley LK, Franceschi RT. Matrix gamma-carboxyglutamic acid protein is a key regulator of pth-mediated inhibition of mineralization in MC3T3-E1 osteoblast-like cells. Endocrinology 2001;142:4379-4388.

33. Hunter GK, Hauschka PV, Poole AR, Rosenberg LC, Goldberg HA. Nucleation and inhibition of hydroxyapatite formation by mineralized tissue proteins. Biochem $J$ 1996;317:59-64.

34. Fujisawa R, Kuboki Y. Affinity of bone sialoprotein and several other bone and dentin acidic proteins to collagen fibrils. Calcif Tissue Int 1992;51:438-442.

35. Fujisawa R, Nodasaka Y, Kuboki Y. Further characterization of the interaction between bone sialoprotein
(BSP) and collagen. Calcif Tissue Int 1995;56: $140-144$.

36. Tye CE, Hunter GK, Goldberg HA. Identification of the type I collagen-binding domain of bone sialoprotein and characterization of the mechanism of interaction. $J$ Biol Chem 2005;280:13487-13492.

37. Gorski JP. Acidic phosphoproteins from bone matrix: A structural rationalization of their role in biomineralization. Calcif Tissue Int 1992;50:391-396.

38. Giannobile WV, Lee CS, Tomala MP, Tejeda KM, Zhu Z. Platelet-derived growth factor (PDGF) gene delivery for application in periodontal tissue engineering. $J$ Periodontol 2001;72:815-823.

39. Anusaksathien $\mathrm{O}$, Jin $\mathrm{Q}$, Zhao $M$, Somerman $M J$, Giannobile WV. Effect of sustained gene delivery of platelet-derived growth factor or its antagonist (PDGF1308) on tissue engineered cementum. J Periodontol 2004;75:429-440.

40. Rutherford RB, Moalli M, Franceschi RT, Wang D, Gu $\mathrm{K}$, Krebsbach $\mathrm{PH}$. Bone morphogenetic proteintransduced human fibroblasts convert to osteoblasts and form bone in vivo. Tissue Eng 2002;8:441-452.

41. Rutherford RB, Racenis P, Fatherazi S, Izutsu K. Bone formation by BMP-7-transduced human gingival keratinocytes. J Dent Res 2003;82:293-297.

42. Jin QM, Anusaksathien O, Webb SA, Rutherford RB, Giannobile WV. Gene therapy of bone morphogenetic protein for periodontal tissue engineering. JPeriodontol 2003;74:202-213.

43. Kirker-Head CA. Potential applications and delivery strategies for bone morphogenetic proteins. Adv Drug Deliu Rev 2000;43:65-92.

44. Franceschi RT, Yang S, Rutherford RB, Krebsbach $\mathrm{PH}$, Zhao M, Wang D. Gene therapy approaches for bone regeneration. Cells Tissues Organs 2004;176: 95-108.

Correspondence: Dr. Sema S. Hakki, Department of Periodontology, Faculty of Dentistry, Selcuk University, 42079 Konya, Turkey. Fax: 90-332-241-0062; e-mail: sshakki@selcuk.edu.tr.

Accepted for publication June 17, 2005. 